\title{
The effect of verbal short term memory on receptive language abilities in bilingual and monolingual children with Specific Language Impairment
}

Ioanna Talli, Stavroula Stavrakaki

Department of Italian, Aristotle University of Thessaloniki, Greece

https://doi.org/10.36505/ExLing-2017/08/0027/000329

\begin{abstract}
The present study investigates whether the bilingual advantage in verbal STM holds for bilingual children with Specific Language Impairment (SLI) and if so whether it affects lexical and syntactic abilities. Sixteen monolingual and 16 bilingual children with SLI, with Greek as L1 and Albanian or Russian as L2 were compared with two control groups of 20 monolingual and 18 bilingual (L1: Greek, L2: Albanian or Russian) TD children in vocabulary, verbal STM and syntactic comprehension. Both clinical groups were worse than mono-TD group. Bi-SLI children were worse than mono-SLI children in vocabulary, in one verbal STM task and in one syntactic comprehension task. The results indicate parallel patterns of deficits in bi- and mono- children with SLI in the domains of STM and syntax.
\end{abstract}

Key words: Specific Language Impairment, verbal STM, syntax, bilingualism.

\section{Introduction}

The term Specific Language Impairment ( $S L I$ ) is used to describe the poor oral language development of a child, which cannot be attributed to neurological, sensorimotor, mental or emotional/ psychological deficits (Leonard, 1998/2014). Difficulties in language development concern mainly the domain of morpho-syntax (Stavrakaki \& van der Lely 2010) and, more specifically, complex structural representations at the levels of syntax and morphology, such as relative clauses (Adani, Forgiarini, Guasti, \& van der Lely 2014; Friedmann, Belletti, \& Rizzi 2009). In addition, children with SLI show deficits in phonological short-term memory (STM) (Newbury, Bishop \& Monaco 2005). Phonological STM is considered a marker for the diagnosis of children with SLI (Archibald \& Gathercole 2006; Conti-Ramsden 2003; Lalioti, Stavrakaki, Manouilidou, \& Talli, 2016) and an indicator of language skills and syntactic comprehension in typically developing children (Rodrigues \& Befi-Lopes 2009).

Recent studies on typically developing bilingual children indicate advantages in the domain of verbal STM (Morales, Calvo, \& Bialystok 2013; Soliman 2014), because they are better in executive functions associated with STM, such as tasks witching (Adi-Japha,

ExLing 2017: Proceedings of 8th Tutorial and Research Workshop on Experimental Linguistics, 19-22 June, Heraklion, Crete, Greece 
Berberich-Artzi, \& Libnawi 2010), which may benefit language acquisition. This study investigates whether the bilingual advantage in verbal STM holds for bilingual children with SLI and if so whether it affects lexical and syntactic abilities. Specifically, our study examines whether there are any differences between monolingual (mono-) and bilingual (bi-) children with SLI on lexical and syntactic skills and on verbal STM.

\section{Methodology}

\section{Participants}

There were four groups of children in total, two clinical groups and two control groups: The clinical groups included sixteen monolingual (mean age 8.11 years) and 16 bilingual children with SLI (mean age 8.4 years), with Greek as a first language (L1) and Albanian or Russian as a second language. These children were diagnosed with SLI by experienced speech and language therapists working in public or private centres for speech and language therapy in Greece. All these primary school aged children were reported to show language delay or disorders at the preschool age and continued to show persistent language deficits at the time of testing. The two control groups included 20 (Greek-speaking) monolingual (mean age 9.0 years) and 18 bilingual (Greek as L1) typically developing children (TD) (mean age 8.6 years) respectively. These children were matched to the two clinical groups in chronological age and nonverbal IQ (Raven's Coloured Progressive Matrices). The control groups did not differ significantly in chronological age and in Raven percentile from the two clinical groups.

\section{Tasks}

Pre-tests included non-verbal IQ assessed with RAVEN Standard Progressive Matrices (Raven 1947) and receptive vocabulary assessed with Peabody Picture Vocabulary Test (Dunn \& Dunn 1981; Greek adaptation: Simos, Sideridis, Protopapas, \& Mouzaki 2011). Experimental tasks included verbal STM, assessed with three tasks: a) a non-word repetition task (a list of 24 three to six syllables non-words; Talli 2010, adapted in Greek from EVALEC Sprenger-Charolles, Colé, Béchennec, \& Kipffer-Piquard 2005), b) a digit span task (forward and backward digit span subtests from Greek WISC-III, Georgas, Paraskevopoulos, Besevegis, \& Giannitsas 1997) c) a sentence repetition task (sentence recall subtest from Diagnostic Test of Verbal Intelligence (DVIQ, Stavrakaki \& Tsimpli 1999). Experimental tasks also included a task of syntactic comprehension consisted of 36 sentences (24 object and subject relative clauses, 4 with reflexive verbs and 8 with passive voice). The examiner would read the sentence and then the child had to select one of the four pictures that 
matched the sentence s/he heard. Percentages of accuracy scores were took into account.

\section{Results}

For the different tasks, ANOVAs were conducted with the 4 groups as the between subject factor. When the effect of group was significant, T-tests were conducted to compare performances of mono-SLI and bi-SLI with mono-TD, between bi-SLI and bi-TD and between the two clinical groups.

Both clinical groups had significantly poorer performance compared to the monolingual TD group in all tasks (except for passives and reflexives for mono-SLI). Bi-SLI children had significantly worse performance than mono-SLI children in vocabulary, in one verbal STM task (sentence repetition) and in one syntactic comprehension task (passives). Comparisons between bi-SLI and bi-TD showed that bi-SLI children were significantly worse than bi-TD children in all tasks (see Table 1).

Table 1. Mean performance (and SDs) of groups on experimental tasks (all

\begin{tabular}{|c|r|r|r|r|}
\hline \hline & Mono-SLI & \multicolumn{1}{c|}{ Bi-SLI } & \multicolumn{1}{c|}{ Mono-TD } & \multicolumn{1}{c|}{ Bi-TD } \\
\hline $\begin{array}{c}\text { Receptive vocabulary } \\
\text { (percentile) }\end{array}$ & $24,9(19,2)$ & $9,9(12,8)$ & $65,8(19,8)$ & $39,2(23,3)$ \\
\hline Digit span (raw score) & $9,6(2,8)$ & $8,4(2)$ & $12,5(2,5)$ & $10,8(2)$ \\
\hline Non-word repetition & $77,6(12,9)$ & $70,6(9,7)$ & $91,6(5,8)$ & $84,2(8,3)$ \\
\hline Sentence repetition & $42,9(2,4)$ & $39,3(6,9)$ & $44,8(0,52)$ & $43,8(1,6)$ \\
\hline Relative clauses & $67,5(16,4)$ & $57,5(14,3)$ & $81,5(10,4)$ & $84,4(5,7)$ \\
\hline Passives & $66,4(26,5)$ & $45,3(25,4)$ & $79,4(17,8)$ & $89,6(18,3)$ \\
\hline Reflexive verbs & $81,3(26,6)$ & $68,8(19,4)$ & $93,7(13,8)$ & $98,6(5,9)$ \\
\hline \hline
\end{tabular}

in percentages, except for the first two).

\section{Discussion - conclusions}

The abovementioned results indicate that deficits in receptive vocabulary are more evident in the domain of receptive vocabulary in bilingual children with SLI than in monolingual ones. In conclusion, the verbal STM does not appear to be superior in children with bi-SLI or to affect their performance neither on syntax nor on vocabulary. These findings highlight the severely impaired status of verbal STM in SLI independently of the number of languages these children are exposed to.

\section{References}

Adani, F., Forgiarini, M., Guasti, M. T., \& van der Lely, H. K. 2014. Number dissimilarities facilitate the comprehension of relative clauses in children with 
(Grammatical) Specific Language Impairment. Journal of Child Language 4l(4), 811-841.

Adi-Japha, E., Berberich-Artzi, J., \& Libnawi, A. 2010. Cognitive flexibility in drawings of bilingual children. Child Development 81, 1356-1366.

Archibald, L., \& Gathercole, S. 2006. Nonword repetition: A comparison of tests. Journal of Speech, Language, and Hearing Research 49, 970-983.

Conti-Ramsden, G. 2003. Processing and linguistic markers in young children with specific language impairment. Journal of Speech, Language and Research 46, $1029-1037$.

Friedmann, N., Belletti, A., \& Rizzi, L. 2009. Relativized Relatives. Types of intervention in the acquisition of A-bar dependencies. Lingua, 119, 67-88.

Georgas, J., Paraskevopoulos, I. N., Besevegis, E. and Giannitsas, N. D. 1997. The Hellenic WISC-III, Athens: Psychometric Laboratory, University of Athens.

Lalioti, M., Stavrakaki, S., Manouilidou C. \& Talli, I. (2016). Subject-Verb Agreement and Verbal Short Term Memory: A Perspective from Greek Children with Specific Language Impairment. First Language, 36, 279-294.

Leonard, B. L. 1998/2014. Children with Specific Language Impairment. Cambridge, MA: MIT Press.

Morales, J., Calvo, A., \& Bialystok, E. 2013. Working memory development in monolingual and bilingual children. Journal of experimental child psychology, 114(2), 187-202.

Rodrigues, A., \& Befi-Lopes, D. M. 2009. Phonological working memory and its relationship with language development in children. Pró-Fono Revista de Atualização Científica, 21(1), 63-68.

Simos, P., Sideridis, G.D., Protopapas, A. \& Mouzaki, A. 2011. Psychometric Characteristics of a Receptive Vocabulary Test for Greek Elementary Students. Assessment for Effective Intervention, 37, 34-50.

Soliman, A. M. 2014. Bilingual advantages of working memory revisited: A latent variable examination. Learning and Individual Differences, 32, 168-177.

Stavrakaki, S., \& van der Lely, H. 2010. Production and comprehension of pronouns by Greek children with specific language impairment. British Journal of Developmental Psychology, 28(1), 189-216.

Talli, I. 2010. Linguistic abilities in Developmental Dyslexia and Specific Language Impairment: A comparative and cross-linguistic approach ( $\mathrm{PhD}$ thesis). Paris Descartes University and Aristotle University of Thessaloniki. 